\title{
The perception of object versus objectless motion
}

\author{
Howard S. Hock • David F. Nichols
}

Published online: 27 February 2013

(C) Psychonomic Society, Inc. 2013

\begin{abstract}
Wertheimer, M. (Zeitschrift für Psychologie und Physiologie der Sinnesorgane, 61:161-265, 1912) classical distinction between beta (object) and phi (objectless) motion is elaborated here in a series of experiments concerning competition between two qualitatively different motion percepts, induced by sequential changes in luminance for twodimensional geometric objects composed of rectangular surfaces. One of these percepts is of spreading-luminance motion that continuously sweeps across the entire object; it exhibits shape invariance and is perceived most strongly for fast speeds. Significantly for the characterization of phi as objectless motion, the spreading luminance does not involve surface boundaries or any other feature; the percept is driven solely by spatiotemporal changes in luminance. Alternatively, and for relatively slow speeds, a discrete series of edge motions can be perceived in the direction opposite to spreading-luminance motion. Akin to beta motion, the edges appear to move through intermediate positions within the object's changing surfaces. Significantly for the characterization of beta as object motion, edge motion exhibits shape dependence and is based on the detection of oppositely signed changes in contrast (i.e., counterchange) for features essential to the determination of an object's shape, the boundaries separating its surfaces. These results are consistent with area MT neurons that differ with respect to speed preference Newsome et al (Journal of
\end{abstract}

Electronic supplementary material The online version of this article (doi:10.3758/s13414-013-0441-1) contains supplementary material, which is available to authorized users.

H. S. Hock $(\square)$

Department of Psychology and Center for Complex Systems and Brain Sciences, Florida Atlantic University, Boca Raton, FL 33431, USA

e-mail: hockhs@fau.edu

D. F. Nichols

Department of Psychology, Roanoke College, Salem,

VA 24153, USA
Neurophysiology, 55:1340-1351, 1986) and shape dependence Zeki (Journal of Physiology, 236:549-573, 1974).

Keywords Beta motion · Phi motion · Edge motion · Luminance-determined motion $\cdot$ Neural mechanisms

The distinction between the perception of object motion and objectless motion has its historical origin in Wertheimer's (1912) phenomenological description of beta and phi motion, which he differentiated according to whether or not the perception of apparent motion for an object that is alternately presented at two spatial locations is accompanied by the perception of the object passing through intermediate spatial positions. The latter is the case for beta, or optimal, motion, which is perceived when the alternation rate is relatively slow, but not for phi motion, which is perceived when the alternation rate is relatively fast. Because the object is not perceived in intermediate positions, phi motion is called "objectless" (Steinman et al. 2000; Wertheimer 1912). ${ }^{1}$ Phi also has been referred to as omega motion (Saucer 1953; Tyler 1973) and shadow motion (Ekroll et al. 2008), in order to denote the impression of an ill-defined "entity" moving across the intermediate space.

The research reported in this article elaborates Wertheimer's (1912) beta/phi distinction by showing that, in addition to their speed dependence, object and objectless motion differ with respect to whether the perceived motion depends on stimulus features that are fundamental to the perception of an object's shape (the boundaries separating the object's surfaces), or instead

\footnotetext{
${ }^{1}$ In contrast with the perceived stationarity of the rapidly alternating visual objects of Wertheimer's (1912) phi motion stimulus, the stimulus for Steinman et al.'s (2000) “magniphi” version is physically stationary. For this stimulus, perceived motion depends entirely on changes in luminance, as is the case in the present study.
} 
is shape invariant, depending only on spatiotemporal changes in "raw" luminance (Chubb \& Sperling, 1989).

\section{Speed preference and intermediate positions}

As we indicated above, Wertheimer's (1912) distinction between relatively slow (beta) and relatively fast (phi) motion is based on whether or not an object is perceived moving through intermediate spatial positions of the apparent motion stimulus. This distinction is also the basis for Sperling and Lu's (1998) account of differences in motion perception for "pedestaled" sine gratings, which are composed of a drifting grating superimposed on a stationary grating with the same spatial frequency. For relatively low temporal frequencies (slow speeds), the pedestal is incorporated into the motion percept, and back-and-forth wobbling motion is perceived; that is, the perception of motion is accompanied by the perception of changes in position for the peaks and troughs of the pedestaled grating. For higher temporal frequencies (faster speeds), the pedestal is not incorporated into the motion percept, and motion is perceived without accompanying changes in perceived position. Sperling and Lu asserted that the latter represents "true objectless motion" (p. 178) because at high speeds, there is no moving object for motion signals to attach themselves to (i.e., the perception of object motion is attributed to a third-order motion system that responds to spatiotemporal changes in feature salience).

\section{Speed preference and shape}

Object motion also can be differentiated from objectless motion on the basis of whether or not the perception of motion depends on the shape of the moving stimulus. Hochberg and Brooks (1978) showed that when two motion paths compete, the path that maintains the shape of the moving object is perceptually selected for relatively long stimulus presentations, but shape correspondences are not a factor for brief stimulus presentations. The high-speed preference of shape-invariant motion correspondences for Hochberg and Brooks is consistent with high-speed motion being shapeless, and therefore objectless.

Also consistent with high-speed motion being shapeless is Burr and Ross's (1982) evidence that contrast sensitivity for drifting sine gratings shifts to lower and lower spatial frequencies with increasing drift speeds. The loss of sensitivity for high and medium spatial frequencies would make it difficult, at best, to discern the shape of an object moving at high speeds.

\section{Blindsight}

Further evidence that high-speed motion perception can be shapeless has been reported for experiments with hemianopes who, as a result of unilateral damage to area V1, have no conscious visual experience and no ability to discriminate the shapes of objects presented to the impaired hemifield. Despite the absence of shape perception, motion direction can be discriminated in that hemifield, but only if it is high-speed motion (Azzopardi \& Cowey 2001; Barbur et al. 1993).

Additional experiments have indicated that direction discrimination in a hemianope's cortically blind hemifield is based on the detection of spatiotemporal changes in luminance, irrespective of any other stimulus feature (Azzopardi $\&$ Hock 2011). These experiments were based on the line motion illusion (previously called polarized gamma motion), which occurs when a surface is present and another surface is presented adjacent to it, or both surfaces are present and one is then removed (Faubert \& von Grünau 1995; Hikosaka et al. 1993a, b; Kanizsa 1978). For unimpaired vision, the shape of the initially presented surface appears to gradually expand (Fig. 1a) or gradually contract (Fig. 1b). In both cases, the outer edge of the shape is perceived as moving through intermediate positions within the appearing or disappearing surface.

Motion determined by these changes in shape can be differentiated from motion determined only by spatiotemporal changes in luminance with a line motion stimulus for which one surface is white and the surface that is presented or removed is black. The presentation and removal of the black surface shifts the centroid of the luminance profile toward and away from the white surface, respectively. As a result, the changes in shape and in luminance profile specify motion in opposite directions (Figs. 1c and 1d). When this stimulus was presented in the hemianope's intact hemifield, Azzopardi and Hock (2011) found that direction judgments were determined by changes in shape. However, when the same stimulus was presented in the hemianope's cortically blind hemifield, where differences in shape could not be discriminated, direction judgments were determined by changes in the luminance profile of the line motion stimulus. Therefore, and as predicted by Sperling and Lu (1998), direction judgments in the hemianope's cortically blind hemifield were objectless: based on spatiotemporal changes in luminance rather than changes in shape.

\section{Edge motion}

The perceived shape of an object depends on the boundaries separating its adjacent surfaces, so the perception of edge motion resulting from changes in contrast at surface boundaries entails features that also determine the object's shape (the first stage within object recognition theories typically begins with the extraction of edges; see, e.g., Biederman 1987; Marr \& Nishihara 1978). Hock and Nichols (2010) showed this with a version of the line motion stimulus that is 
Fig. 1 Different versions of stimuli that induce the line motion illusion. (a-d) Stimuli that provided evidence that motion direction discrimination is based on changes in

luminance rather than shape for a patient who has cortical damage that eliminates object and shape perception in one hemifield (Azzopardi \& Hock 2011). (e, f) Stimuli inducing the line motion illusion by changing the luminance for one of two adjacent surfaces (Hock \& Nichols 2010). The vertical arrows indicate whether edge contrast decreases or increases during Frame 2. Motion is in the counterchange-specified direction, as determined by oppositely signed changes in edge contrast, starting at the surface boundary where edge contrast decreases and ending at the surface boundary where it increases
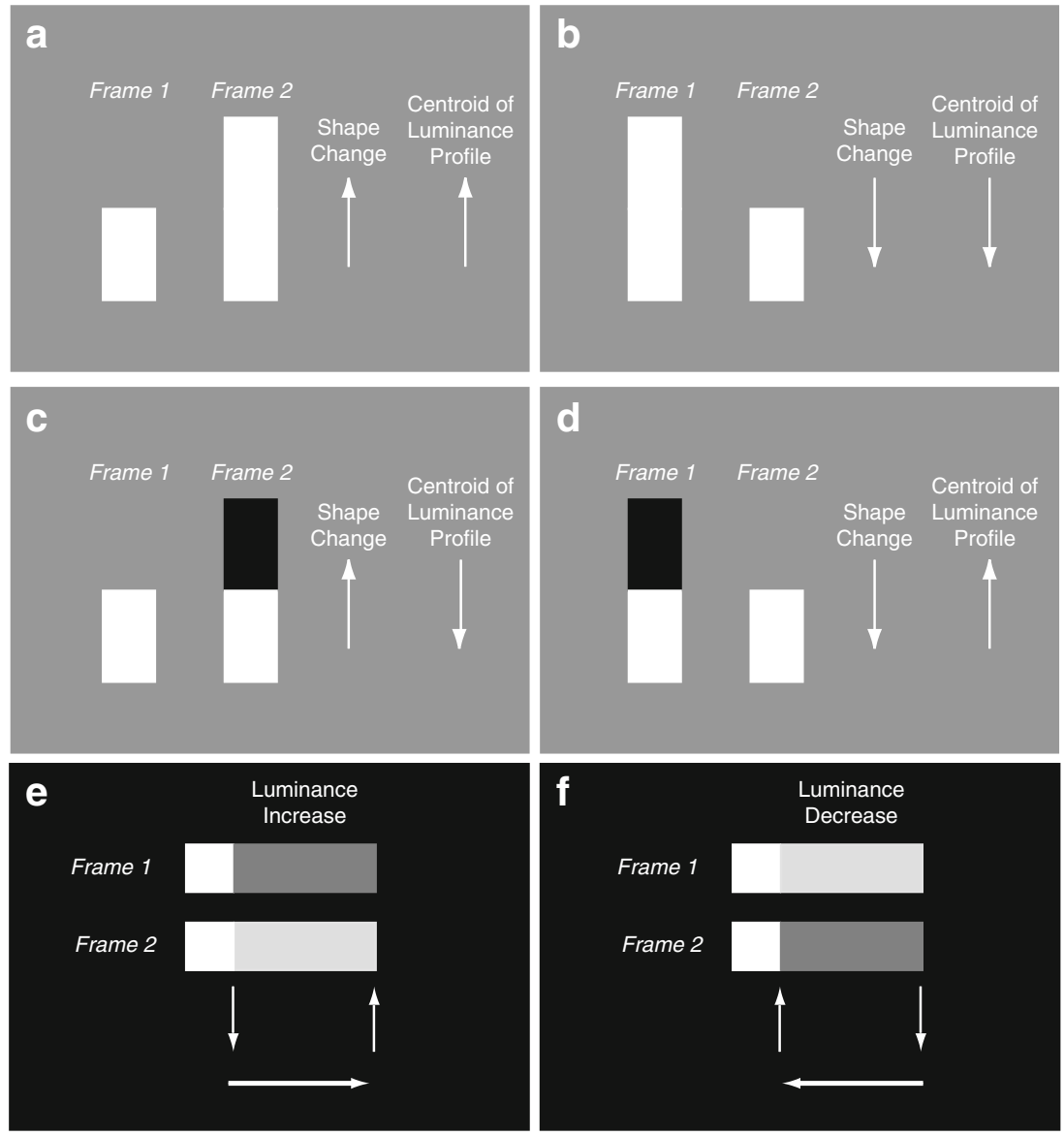

the basis for the present study. In this version, luminance is changed for one of two adjacent, simultaneously visible surfaces. Luminance increases result in the perception of edge motion across the changing surface, away from the adjacent, unchanging surface (Fig. 1e). Luminance decreases result in the perception of edge motion in the opposite direction (Fig. 1f). For both increases and decreases in the surface's luminance, edge motion is perceived when there are oppositely signed changes in contrast (i.e., counterchange; Hock et al. 2002; Hock et al. 2009). It starts at the boundary of the changing surface, where edge contrast decreases, and ends at the surface's opposite boundary, where edge contrast increases. In a manner analogous to classical beta motion, the perceptually moving edge appears to occupy a succession of intermediate positions as it crosses the changing surface.

\section{The edge and spreading-luminance motion percepts}

The stimuli tested in the experiments that follow are two-dimensional geometric objects composed of six simultaneously visible rectangular surfaces that form a chain of line motion stimuli, none of which is displaced. When the rectangles are adjacent and frame durations are relatively long, as in Movie 1, a discrete series of edge motions is perceived in the direction opposite from the sequence of luminance changes. ${ }^{2}$ The edges appear to move through intermediate positions within the object's changing surfaces, as in Hock and Nichols (2010), with the locus of the leftward edge motions shifting to the right from one stimulus frame to the next. However, for the same stimulus but with briefer frame durations, the spreading-luminance motion is perceived as continuously sweeping across the object in the rightward direction (Movie 2). The "entity" that appears to be moving is phenomenologically shapeless: There is no impression of motion within the changing rectangular surfaces, and no involvement in the motion percept of the surfaces' boundaries or of any feature other than luminance. 


\section{Study objectives}

The psychophysical experiments that follow varied in frame durations, the widths of the rectangles, whether the rectangles were vertically or horizontally elongated, and whether or not thin gaps separated the rectangles. The objectives were to (1) determine whether the edge motion and spreading-luminance motion percepts described above differ in their speed and/or shape dependence, (2) confirm that the perception of edge motion depends on the presence of oppositely signed changes in edge contrast (i.e., counterchange), and (3) show that attentive feature tracking (Cavanagh 1992) is not required for either motion percept.

\section{Experiment 1}

Frame duration and the width of the rectangles composing the six-rectangle stimulus were varied in order to determine whether they would differentially affect the spreadingluminance and edge motion percepts, which were in opposing directions.

\section{Method}

Stimuli The test stimulus was composed of six adjacent rectangles, all of which were simultaneously visible. The left-to-right gradient of luminance values was positive for half of the trials (the higher luminance values were on the right, as in Fig. 2) and negative for the other half of the trials (the higher luminance values were on the left, which is not shown). The background luminance was $0 \mathrm{~cd} / \mathrm{m}^{2}$.

Motion percepts were induced by sequentially changing the luminance of the rectangles (from left to right or from right to left) over a succession of six frames. (The luminance values for the positive gradient are indicated within each rectangle in Fig. 2.) The motion-inducing changes in luminance for the stimuli with a positive luminance gradient were as follows: During Frame 2, the leftmost two rectangles increased in luminance, with the luminance increase being greater for the left-hand member of the pair. During Frame 3, luminance increased for the rectangles that were second and third from the left, with the increases again being greater for the left-hand member of the pair, and so on through Frame 6. Rightward spreading-luminance motion was specified by this directional sequence of luminance changes.

A series of edge motions in the opposite, leftward direction were specified by the same luminance changes. When luminance increased for the leftmost pair of rectangles during Frame 2 , a pattern of counterchanging contrast was created by oppositely signed changes in edge contrast at the boundaries of the
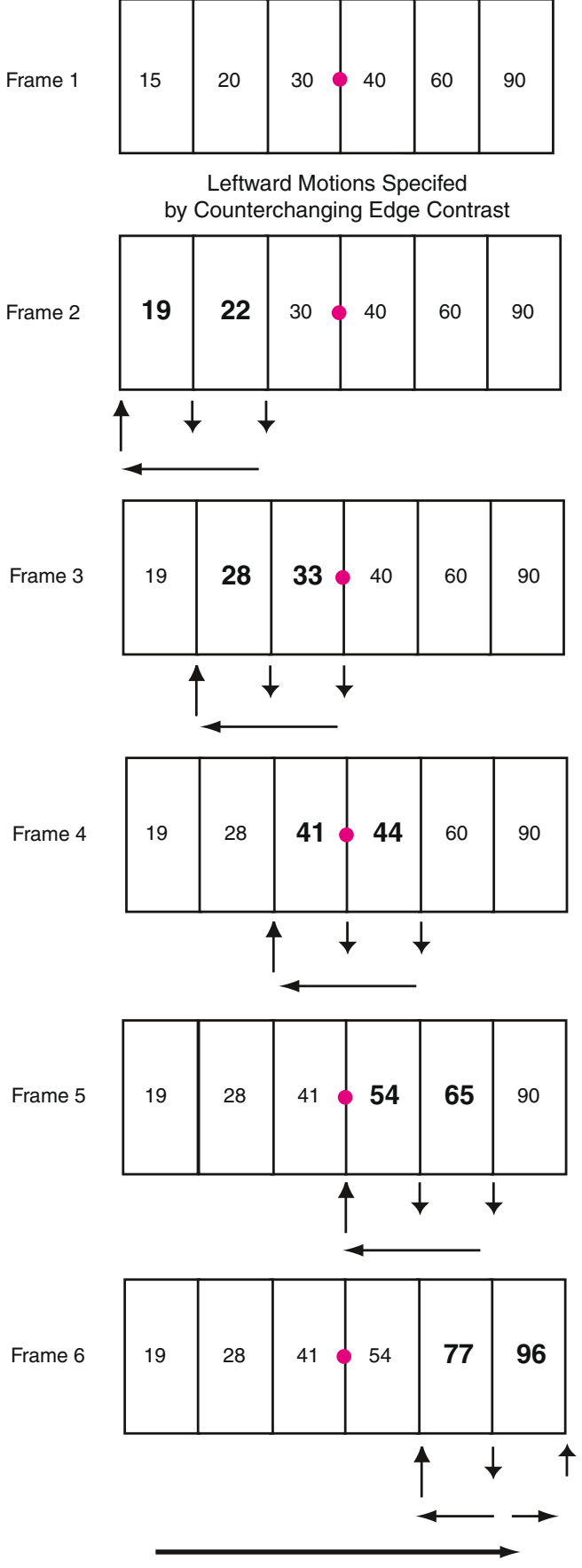

Rightward Spreading-Luminance Motion

Fig. 2 Sample stimulus for Experiment 1. Luminance values, expressed in units of candles per square meter, are indicated within each of six adjacent rectangles. Indicated in bold are the luminance increases for pairs of adjacent rectangles that occur during successive frames. The luminance increases create either rightward spreadingluminance motion across the entire stimulus or a discrete succession of leftward motions signaled by the counterchange of edge contrast at the boundaries of the changing surfaces. The directions of the vertical arrows indicate decreases and increases in edge contrast; their lengths indicate the relative size of the contrast change. The locus of the leftward motions signaled by counterchanging edge contrast shifts to the right during successive frames (the opposite is the case for the mirror-image reversed version of the stimulus) 
changing rectangles (Fig. 2). During Frame 3, the locus of these counterchange-specified leftward edge motions shifted to the right, as it did for all frames through Frame $6{ }^{3}$ The directions of the edge motion and spreading-luminance motion percepts were reversed for the stimuli with a negative luminance gradient (luminance changes began with the rightmost rectangles).

The stimuli were centered in the screen of an NEC MultiSync FP 955 monitor and viewed from a distance of $30 \mathrm{~cm}$, which was maintained with a head restraint. From this distance, the width of the rectangles intercepted visual angles of $0.50,0.84$, and $1.33 \mathrm{deg}$. Their height was $5.3 \mathrm{deg}$. A red fixation dot $(0.16 \times 0.16 \mathrm{deg})$ was presented in the center of the stimulus. The first and last frames of each sixframe trial were 1,600 and $400 \mathrm{~ms}$ in duration, respectively, and the durations of Frames 2-5 varied among 33, 50, 67, $84,100,117,134$, and $150 \mathrm{~ms}$.

Design Blocks of 80 trials were determined by orthogonal combination of the eight frame durations, two luminance gradients (positive and negative), and five repetitions. Trial order was randomized within subblocks of 16 trials. Three blocks of 80 trials were presented during each testing session, one block for each rectangle width. Their order was counterbalanced over the six testing sessions.

Participants Four students at Florida Atlantic University voluntarily participated in this experiment. All were naïve with respect to its purpose.

Procedure The participants were instructed to maintain fixation on the red dot in the center of the stimulus, and to avoid having their attention drawn to the initial luminance changes on either the left or the right end of the six-rectangle stimulus and then tracking luminance changes across the stimulus. They pressed keys on the computer keyboard to indicate whether or not they had perceived motion at any time during the just-ended stimulus presentation, and if they had seen motion, whether it was toward the right or the left. They reported "no motion" when they saw only a sequence of disconnected luminance increments.

\section{Results}

Directional motion was perceived during almost every trial. The relative frequencies of the spreading-luminance and edge motion percepts depended on both frame duration

\footnotetext{
${ }^{3}$ With the exception of the far left edge of the rectangles, with a positive luminance gradient, and the far right edge of the rectangles, with a negative luminance gradient, the pattern of counterchange was the same when edge contrasts were calculated as Michelson contrasts.
}

and rectangle width. For each participant, the perceived motion was predominantly in the spreading-luminance direction for brief frame durations (Fig. 3a), and predominantly in the edge motion direction for longer frame durations (Fig. 3b). The effect of frame duration on the proportions of motion-perceived trials in both the spreading-luminance direction and its complement, motion-perceived trials in the edge motion direction, was statistically significant, $F$ $(7,72)=93.26, p<.0001$. The effect of rectangle width also was statistically significant for both types of motion, $F(2$, 72) $=10.26, p<.0002$.

For intermediate frame durations, either spreadingluminance motion or edge motion could be perceived for the same stimulus. This evidence for bistability indicated that the opposing motion directions competed: One predominated while the other was suppressed. For this reason, the effects of frame duration and rectangle
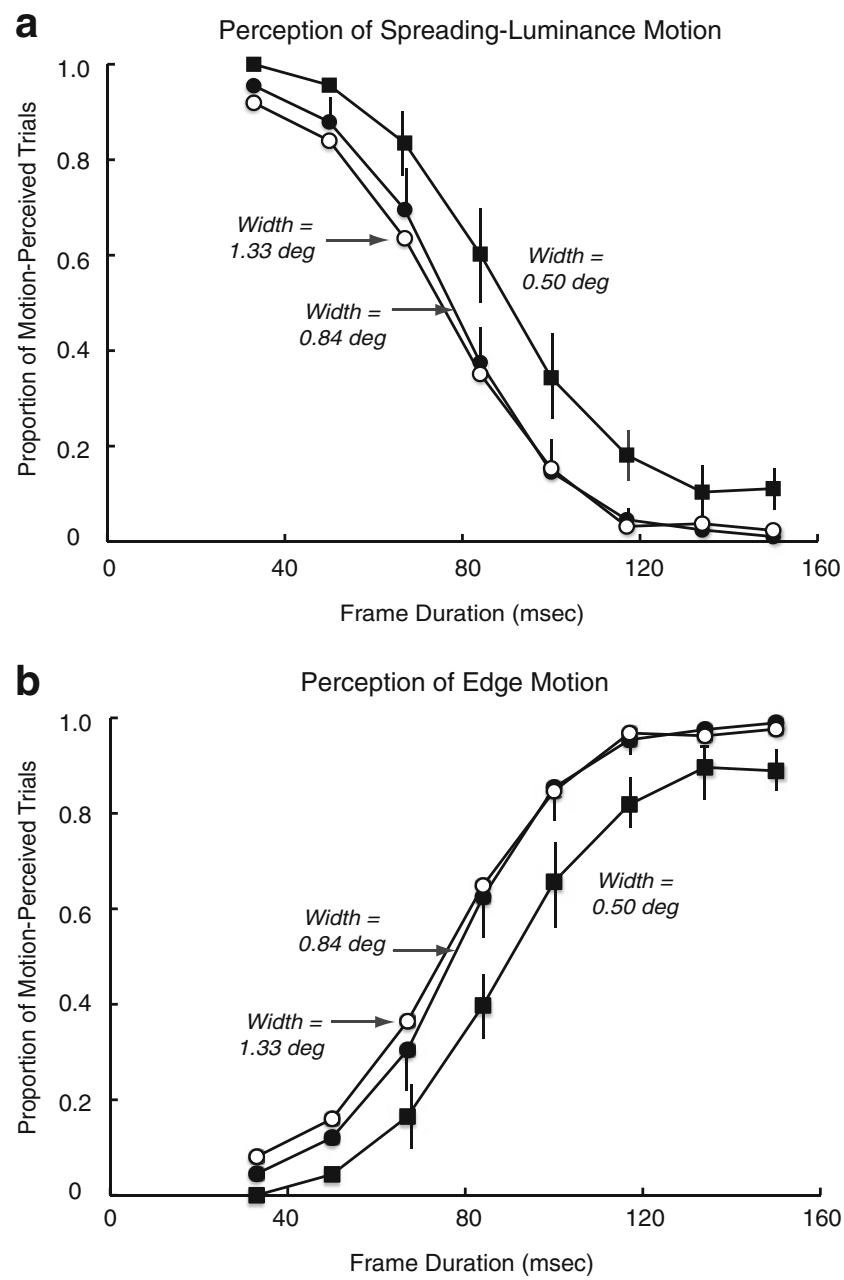

Fig. 3 Results for Experiment 1: Effects of frame duration and rectangle width on the perception of motion in the (a) spreading-luminance direction and (b) edge motion direction (averaged over the four participants). Each of the means is based on 160 trials (40 trials for each of the four participants). Vertical lines attached to the markers denote the average of the participants' standard errors of the means 
width observed for motion in the spreading-luminance direction might have been due to inhibitory competition from the speed- and width-dependent motion in the opposing edge motion direction, or vice versa. This uncertainty was resolved in Experiment 2, whose additional purpose was to confirm that the presence of counterchanging edge contrast is necessary for the perception of edge motion.

\section{Experiment 2}

This experiment differed from Experiment 1 in only one respect: The rectangles were separated by thin, 0.16-deg gaps. It was expected that the perception of edge motion would be eliminated because all changes in edge contrast would now be same-signed (Fig. 4a). However, the luminance values of the surfaces were changed exactly as in Experiment 1, so it was expected that spreading-luminance motion would be perceived, despite the presence of the gaps. (This is confirmed by Movie 3.) By thus isolating the perception of spreadingluminance motion, any effects of frame duration and/or rectangle width would not be attributable to inhibitory competition from the edge motion percept. The participants from Experiment 1 also participated in this experiment.

\section{Results}

As anticipated, the insertion of gaps between the rectangles eliminated the perception of edge motion, as counterchanging edge contrast was no longer present. Thus, only spreading-luminance motion was perceived. As in Experiment 1, this motion was perceived most often for relatively brief frame durations (Fig. 4b). For longer frame durations, a disconnected sequence of luminance increments rather than edge motion, was perceived (Movie 4). The effect of frame duration was statistically significant, $F(7,72)=30.20, p<.0001$, confirming the high-speed preference of motion determined by spatiotemporal changes in luminance. The possibility that this effect was the result of inhibitory competition from the opposing edge motion could be ruled out, because the latter was not a factor in this experiment.

Rectangle width did not affect the perception of spreading-luminance motion, $F(2,72)<1.0$, indicating that its apparent effect on spreading-luminance motion in Experiment 1 had been due to inhibitory competition from the directionally opposing edge motion percept, and conversely, that the effect of rectangle width on
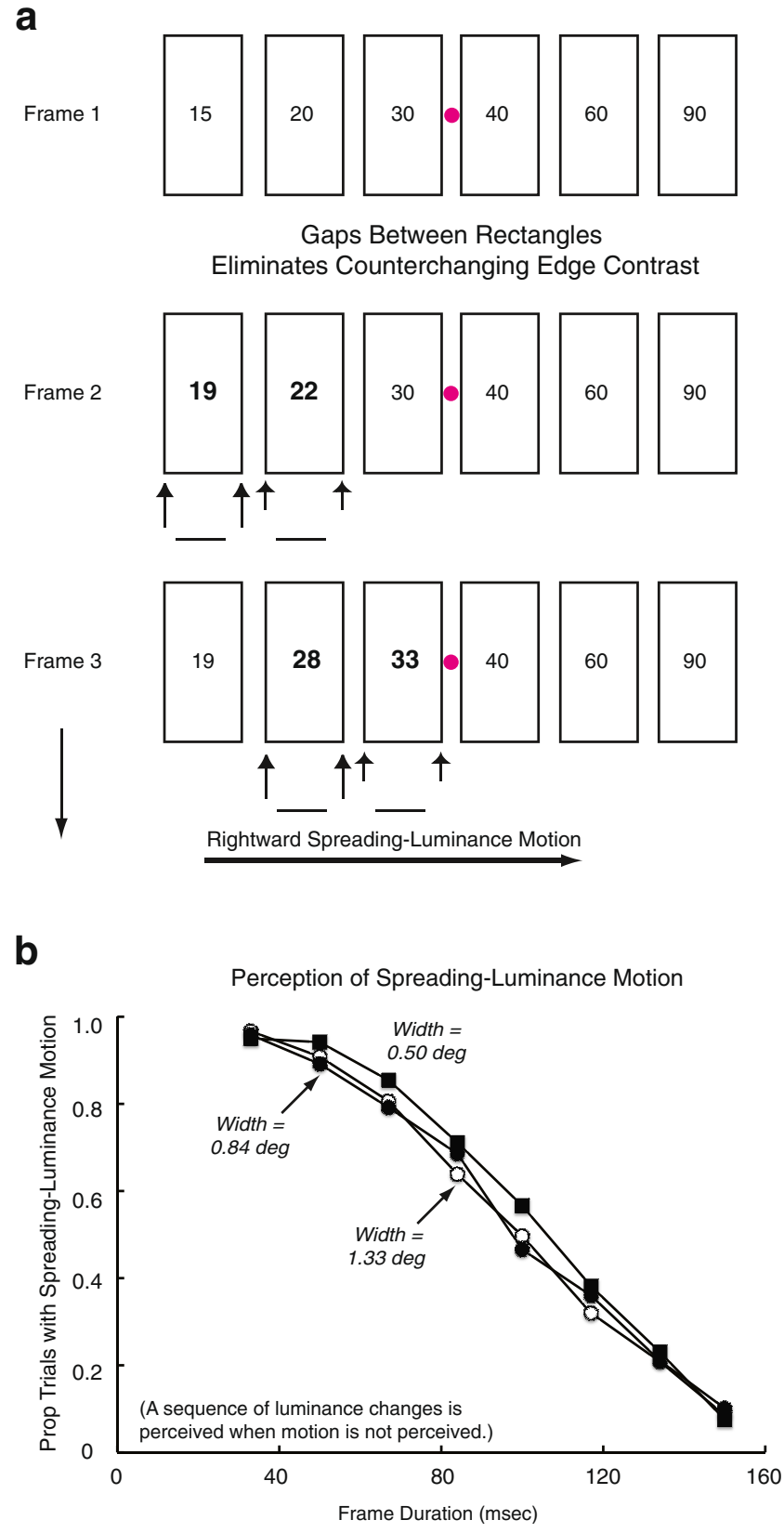

Fig. 4 Stimuli and results for Experiment 2. (a) The same six-rectangle stimuli as in Experiment 1, with the exception that 0.16-deg gaps are inserted between the rectangles. As indicated by the directions of the vertical arrows, all the changes in contrast at the surface boundaries are same-signed, eliminating counterchanging edge contrast. (b) Experimental results indicating the perception of motion in the spreadingluminance direction (averaged over the four participants). Edge motion was not perceived. Each of the means is based on 160 trials (40 trials for each of the four participants)

the perception of edge motion in Experiment 1 was not due to inhibitory competition from the directionally opposing spreading-luminance motion (the latter was not affected by rectangle width when isolated in Exp. 2). The effect of rectangle width resulted from the reduced perception of edge motion for the narrowest rectangles 
(0.50-deg width), possibly because they provided a relatively small spatial "window" for perceiving an edge moving across intermediate positions within the rectangles. Supporting this possibility was the observation in a preliminary experiment that the perception of edge motion was almost never reported when the rectangles were $0.16 \mathrm{deg}$ wide.

It might be argued that the motion in what we have identified as the direction specified by counterchanging edge contrast in Experiment 1 was due instead to the detection of local spatiotemporal changes in luminance. This was possible because with each frame change, luminance increased simultaneously but unequally for pairs of adjacent rectangles. For the stimulus with a positive luminance gradient, the change in luminance was greater for the left- than for the right-hand rectangle of each pair, so the centroid of the locally changing luminance profile shifted to the left. Arguing against this possibility is the fact that the luminance changes were the same, regardless of whether or not there were gaps between the rectangles. If edge motion were determined by these shifts in the centroid of the luminance profile, it also would have been perceived in Experiment 2, despite the presence of the gaps.

\section{Experiment 3}

Placing gaps between the rectangles in Experiment 2 changed the shape of the stimulus. What had been a single geometric object composed of six rectangular surfaces became six objects, each composed of a single rectangular surface. The change in shape eliminated the perception of edge motion, but spreading-luminance motion continued to be perceived. This indication that the edge and spreading-luminance motion percepts might differ with respect to their shape dependence was directly tested in this experiment by varying the shape of the rectangles composing the six-rectangle stimulus: They were either vertically or horizontally elongated.

\section{Method}

The horizontally elongated rectangles were $3.4 \mathrm{deg}$ wide and $1.33 \mathrm{deg}$ high, and the vertically elongated rectangles, which were matched in area, were $0.84 \mathrm{deg}$ wide and $5.3 \mathrm{deg}$ high. The luminance, frame structure, and procedure were the same as in Experiment 1. Two new participants, students at Florida Atlantic University who were naïve with respect to the purpose of the experiment, were tested with eight blocks of 80 order-randomized trials: four blocks with the vertically elongated, and four with the horizontally elongated rectangles.

\section{Results}

Directional motion was perceived during almost every trial. For both participants, the perceptions of spreading-luminance motion and edge motion were differentiated by frame duration and the shape of the rectangles. As before, the perceived motion was predominantly in the spreading-luminance direction for brief frame durations, and predominantly in the edge motion direction for longer frame durations. Most importantly, both participants perceived spreading-luminance motion more often for the horizontally elongated rectangles (Fig. 5a), and perceived edge motion more often for the vertically elongated rectangles (Fig. 5b). As was the case in the results of Experiment 1 , it was uncertain whether the effect of shape for one of the percepts was due to inhibitory competition from the other percept, which was in the opposite direction. That is, the effect of shape on spreading-luminance motion might in fact have been the result of inhibitory competition from shape-dependent edge motion, or vice versa. This was resolved in Experiment 4.

\section{Experiment 4}

This experiment was identical to Experiment 3, including the testing of the same participants. The only difference was the 0.16-deg gaps that were inserted between the rectangles. As in Experiment 2, the purpose of the gaps was to isolate the perception of spreading-luminance motion.

\section{Results}

Inserting gaps between the rectangles again eliminated the edge motion percept, leaving only the perception of spreading-luminance motion, and again, only for relatively brief frame durations. For both participants, the spreadingluminance motion percept exhibited shape invariance; it was unaffected by whether the rectangles were vertically or horizontally elongated (Fig. 5c). This meant that the perception of edge motion had been affected by shape in Experiment 3; the apparent effect of shape on spreadingluminance motion was a consequence of inhibition from the directionally opposing edge motion percept. The stronger edge motion perceived for the vertically than for the horizontally elongated rectangles in Experiment 3 could have been the result of the changes in contrast being greater for longer vertical edges, and/or the edge motion being more strongly perceived over the much smaller horizontal distance. 
Fig. 5 (a, b) Results for Experiment 3: Effects of frame duration and shape of the rectangles (whether they were vertically or horizontally elongated) on the perception of motion in the (a) spreadingluminance direction and (b) edge motion direction. (c) Results for Experiment 4 (with gaps inserted between the rectangles): Effects of frame duration and shape of the rectangles (whether they were vertically or horizontally elongated) on the perception of spreading-luminance motion. Edge motion was not perceived. Results in the graphs are averaged over the two participants. Each of the means is based on 80 trials ( 40 trials for each of the two participants). Vertical lines attached to the markers in the graphs denote the average of the participants' standard errors of the means
EXPERIMENT 3: NO GAPS BETWEEN THE RECTANGLES
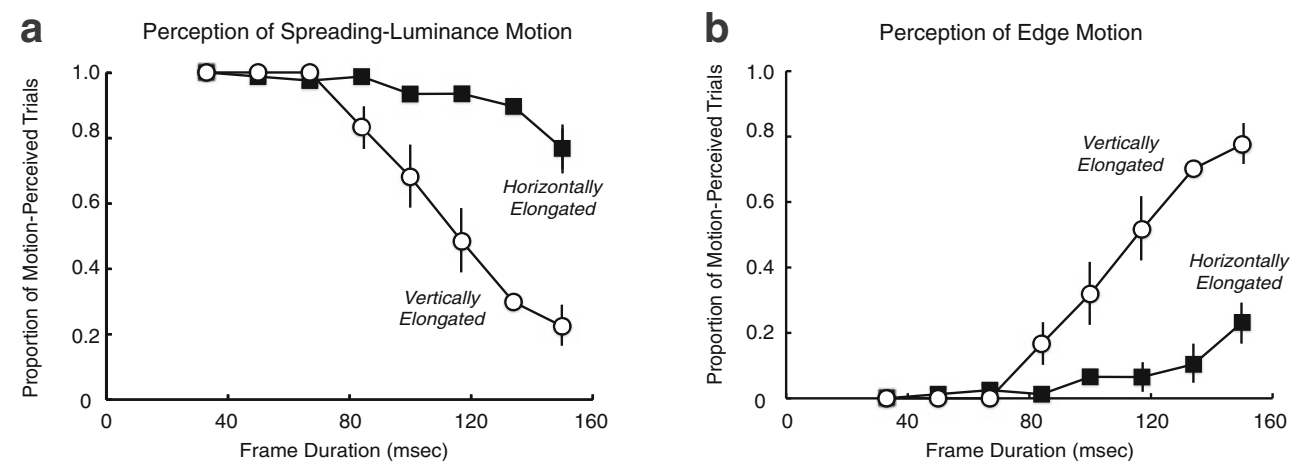

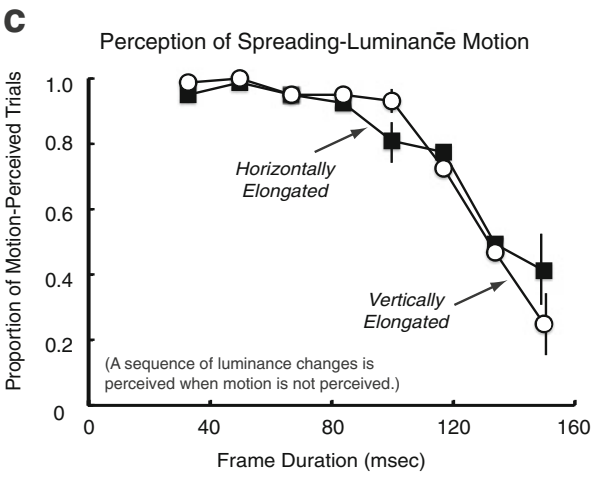

\section{Experiment 5}

Participants in the preceding experiments had been instructed to maintain attention on the fixation dot in the center of the six-rectangle stimulus, and to suppress any tendency to attentively track changes in luminance (Cavanagh, 1992). The purpose of this experiment was to confirm that attentive tracking was not required for the perception of either spreading-luminance motion or edge motion.

\section{Part 1: Spreading-luminance motion}

\section{Method}

A six-rectangle stimulus with the same luminance values as in the preceding experiments was presented. Gaps were placed between the rectangles in order to eliminate the possibility that edge motion would be perceived. The participants, three students from Florida Atlantic University who were naïve with respect to the purpose of the experiment, were instructed to maintain their attention on the fixation dot in the center of the stimulus, and to avoid tracking the sequential changes in luminance. The rectangles were $5.3 \mathrm{deg}$ high and $3.16 \mathrm{deg}$ wide. The gaps were 0.16 deg wide, and the only frame duration tested was $33 \mathrm{~ms}$. If attentive tracking were responsible for the perception of motion, it would have been at a rate of $100 \% \mathrm{~s}(3.16+0.16 \mathrm{deg} / 33 \mathrm{~ms})$, twice its estimated upper limit (Verstraten et al. 2000).

\section{Results}

Despite its speed being out of the range for attentive tracking, the perception of motion in the spreading-luminance direction was reported for an average of $99.6 \%$ of the 40 trials per participant (20 with the positive and 20 with the negative luminance gradient). It was concluded that attentive tracking is not required for the perception of spreading-luminance motion.

\section{Part 2: Edge motion}

\section{Method}

The six-rectangle stimulus was composed of rectangles $5.3 \mathrm{deg}$ high and 0.84 deg wide that were not separated by gaps. In contrast with the preceding experiments, only two frames were presented per trial. Following the 1,600-ms first frame, 
luminance changes occurred for two adjacent rectangles during Frame 2, which was $400 \mathrm{~ms}$ in duration. The luminance values of the rectangles (second and third from the left or second and third from the right), which are presented in Fig. 6, corresponded to the values for the second and third frames in the preceding experiments. Without a multiframe sequence of luminance changes, the perception of spreading-luminance motion was eliminated. Only edge motion was perceived.

The location of the fixation dot was varied, so that if the motion were due to attentive tracking, it would be away from the fixation dot. For half of the 80 trials, the fixation dot was in the center of the stimulus, so that the counterchange-specified edge motion and the motion attributable to attentive tracking would be in the same direction (Figs. 6a and b). For the other half, the fixation dot was located between the first and second rectangles from the left (Fig. 6c) or the right (Fig. 6d), so that the counterchange-specified motion and the motion attributable to attentive tracking would be in opposite directions.

\section{Results}

The results, averaged over the same three participants as in the first part of the experiment, indicated that edge motion was perceived in the direction codetermined by counterchange and attentive tracking for $99.2 \%$ of the trials with a central fixation dot, and in the direction determined by counterchange, but not by attentive tracking, for $99.0 \%$ of the trials in which these processes predicted motion in opposite directions (when the fixation dot was between the two outermost rectangles). It was concluded from the latter result that attentive tracking is not necessary for the perception of edge motion.

\section{Discussion}

The experiments reported in this article were based on a two-dimensional geometric object for which two qualitatively different motions were perceived: spreadingluminance motion and edge motion. Spreading-luminance motion was best perceived for relatively brief frame durations (fast speeds), and exhibited shape invariance. That is, it was perceived regardless of whether or not gaps separated the rectangles, and was unaffected by whether the rectangles were vertically or horizontally elongated. There was no impression of motion within the changing surfaces of the stimuli, and no involvement in the motion percept of the
Fig. 6 Stimuli for the second part of Experiment 5. Presented are the six-rectangle stimuli with positive and negative luminance gradients. The fixation dots are either in the center of the stimulus (motion that would result from attentive tracking is in the same direction as the motion specified by counterchanging edge contrast) or offset (motion that would result from attentive tracking is in the opposite direction to the motion specified by the counterchanging edge contrast)

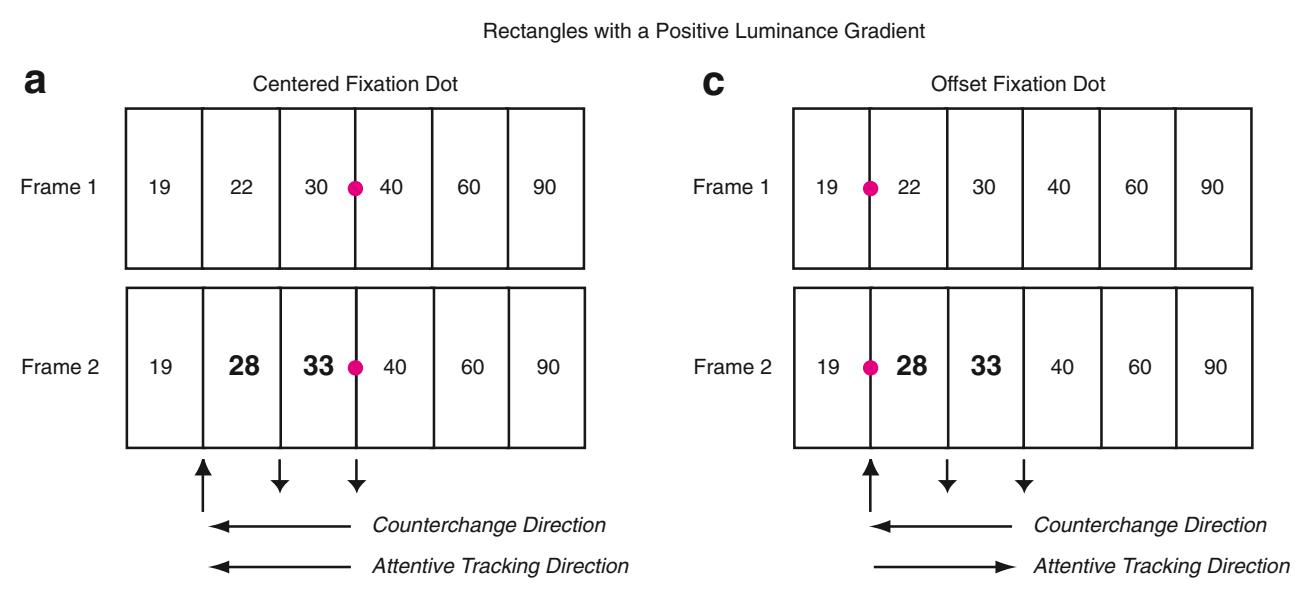

Rectangles with a Negative Luminance Gradient

b

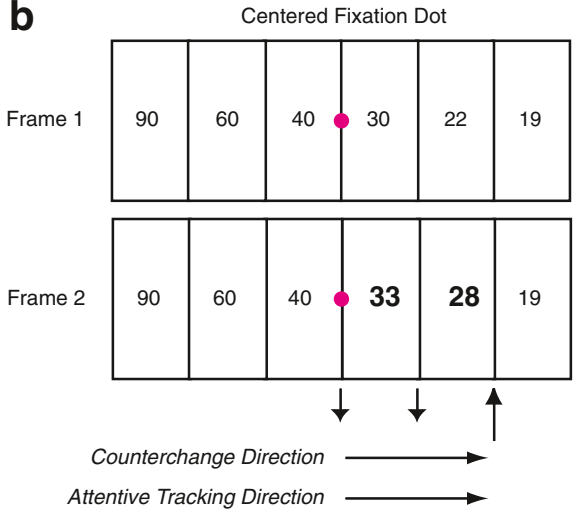

d

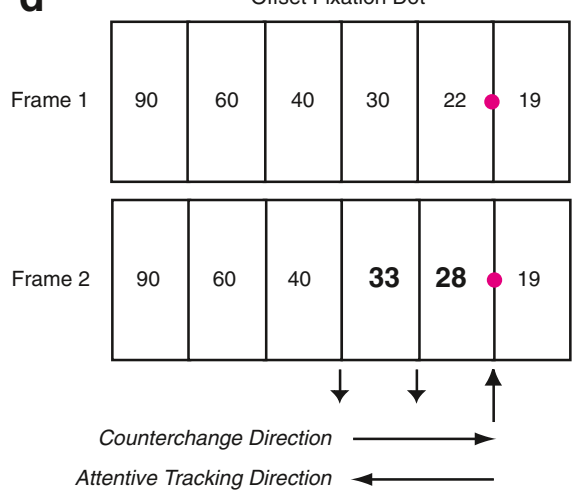


surfaces' boundaries or any feature other than luminance. Like the objectless motion perceived at high temporal frequencies for Sperling and Lu's (1998) pedestaled gratings, and the phi/omega/shadow motion perceived for rapidly alternating apparent-motion stimuli (Ekroll et al. 2008; Tyler 1973; Wertheimer 1912), the spreading-luminance percept entails a rapidly moving "entity" that is phenomenologically shapeless.

Edge motion was best perceived for relatively long frame durations (slower speeds). It starts at the boundaries of surfaces that are changing in luminance, and akin to beta motion, the perceptually moving edges appear to pass through intermediate positions within the surfaces before ending at their opposite boundaries. Moreover, the edge motion percept exhibited shape dependence: It was eliminated by the insertion of gaps between the rectangles, and was best perceived when the rectangles composing the stimulus were vertically rather than horizontally elongated. The shape dependence of the edge motion percept is consistent with edges/boundaries being fundamental to the perception of an object's shape, so the perception of edge motion is reflective of what would be expected for the perception of object motion in general. Whether shape dependence is limited to variations in shape that affect the detection of counterchanging contrast remains to be determined.

Shape-dependent motion processing also has been observed by Edwards (2009). He showed that the discrimination of motion direction is enhanced for a group of four coherently moving signal dots embedded in a field of randomly moving noise dots when the signal dots form a fixed, well-defined spatial pattern (e.g., a square), as compared with when their arrangement is variable. Because mixing the luminance polarity of the signal dots adversely affected motion direction discrimination only when the dots formed well-defined patterns, Edwards argued for the possibility of separate form-specific and "standard" motion systems. It is tempting, given the results of the present study, to speculate that such a form-specific system is the basis for the perception of object motion, whereas the "standard" motion system is the basis for the perception of objectless, luminancedetermined motion.

\section{Neural pathways}

The differences in speed preference and shape dependence for object and objectless motion are consistent with the perception of beta motion depending on neurons tuned to slow speeds and high spatial frequencies, and the perception of phi motion depending on neurons tuned to fast speeds and low spatial frequencies (Burr \& Ross 1982). Although the suggestion is speculative, it is conceivable that object and objectless motion engage different neural pathways to area MT, a cortical area comprising motion-sensitive neurons that are almost exclusively directionally selective. (Newsome et al. 1986) have shown, for slow apparent motion speeds, that spatial separation affects human motion/nonmotion thresholds and has corresponding effects on directional selectivity for both V1 and MT neurons of the macaque. For faster speeds, however, the psychophysical and neural effects of spatial separation correspond for MT but not V1 neurons. This indication that high-speed motion does not rely on V1 processing implies that motion information is reaching MT through the superior colliculus rather than V1. Confirming this possibility is evidence that the cooling or lesioning of V1 leaves a high proportion of macaque MT neurons active, and that the additional destruction of the superior colliculus completely eliminates MT activation (Rodman et al. 1989, 1990). Given our results indicating the high-speed preference of motion perception based exclusively on spatiotemporal changes in luminance, it is conceivable that the collicular route to MT is the primary pathway for objectless motion perception. Supporting this possibility is evidence that hemianopes with unilateral damage to V1 can discriminate the direction of high-speed motion in their cortically blind hemifield, despite the absence of object/shape discrimination (Azzopardi \& Cowey 2001; Barbur et al. 1993), and further, that this direction discrimination is based on spatiotemporal changes in luminance rather than changes in shape (Azzopardi \& Hock 2011).

The existence of different neural pathways to area MT, with different preferences for slow versus fast motion, implies that its neurons might differ with respect to whether they are involved in the perception of object or objectless motion. Such a difference was observed in studies indicating that the responses of some directionally selective MT neurons are affected by the shape of moving stimuli, whereas others are not so affected (Albright 1992; Zeki 1974). In addition, (Lamme et al. 1999) found that the boundary and surface information necessary for the perception of both an object's motion and its shape are encoded in area V1. All of these facts are consistent with (1) a neural pathway for the perception of object motion that proceeds from V1 to shape-dependent MT neurons, and perhaps on from there to neurons in the lateral occipital cortex that combine motion and shape information for the perception of 3-D objects (Murray et al. 2003), and (2) a neural pathway for the perception of objectless motion that proceeds from the superior colliculus to shape-invariant MT neurons (bypassing $\mathrm{V} 1$ ), and perhaps on to MSTd neurons that process optic flow.

\section{Conclusions}

The results reported in this article combined phenomenology and psychophysics to elaborate Wertheimer's (1912) 
classical distinction between beta (object) motion and phi (objectless) motion. His account of beta motion is more strongly linked to the perception of object motion by evidence that edge motion is accompanied by the phenomenological impression that the edge is passing through intermediate spatial positions as it is perceived moving across a changing surface, and by psychophysical evidence that edge motion prefers slow speeds and exhibits shape dependence. The significance of showing this for edge motion is that the motion is based on changes in contrast for the same features that are essential to the determination of an object's shape: the boundaries separating the object's surfaces. Indeed, it is likely that the binding of an object's shape and its motion is facilitated by their perception depending on such common features.

Wertheimer's (1912) account of phi motion is more strongly linked to the perception of objectless motion by the phenomenological impression that spreading-luminance motion-like phi, omega, and shadow motion-entails a moving "entity" that is shapeless, and by psychophysical evidence indicating that spreading-luminance motion prefers high speeds and exhibits shape invariance. The significance of showing this for spreading-luminance motion is that this motion percept is based only on spatiotemporal changes in luminance, with no involvement in the motion percept of the surfaces' boundaries or of any feature other than luminance.

Finally, the results reported in this article were obtained with stimuli for which the motion determined by counterchange and the motion determined by spatiotemporal changes in luminance were in opposing directions. In research that is underway, we examine these alternative informational bases for the perception of object and objectless motion with stimuli in which they do not compete, with the further objective of distinguishing between the neural pathway for object motion, which is thought to pass through V1 en route to MT, and the neural pathway for objectless motion, which is thought to pass through the superior colliculus, bypassing V1 en route to MT.

Author Note We thank Paul Azzopardi for valuable suggestions following his careful reading of an earlier version of the manuscript.

\section{References}

Albright, T. D. (1992). Form-cue invariant motion processing in primate visual cortex. Science, 255, 1141-1143.

Azzopardi, P., \& Cowey, A. (2001). Motion discrimination in cortically blind patients. Brain, 124, 30-46.
Azzopardi, P., \& Hock, H. S. (2011). Illusory motion perception in blindsight. Proceedings of the National Academy of Sciences, 108, 876-881.

Barbur, J. L., Watson, J. D. G., Frackowiak, R. S. J., \& Zeki, S. (1993). Conscious visual perception without V1. Brain, 116, 1293-1302.

Biederman, I. (1987). Recognition-by-components: A theory of human image understanding. Psychological Review, 94, 115-147. doi:10.1037/0033-295X.94.2.115

Burr, D. C., \& Ross, J. (1982). Contrast sensitivity at high velocities. Vision Research, 22, 479-484.

Cavanagh, P. (1992). Attention-based motion perception. Science, 257, 1563-1565. doi:10.1126/science. 1523411

Chubb, C., \& Sperling, G. (1989). Two motion perception mechanisms revealed through distance-driven reversal of apparent motion. Proceedings of the National Academy of Sciences, 86, 29852989.

Edwards, M. (2009). Common-fate motion processing: Interaction of the On and Off pathways. Vision Research, 49, 429-438. doi:10.1016/j.visres.2008.11.010

Ekroll, V., Faul, F., \& Golz, J. (2008). Classification of apparent motion percepts based on temporal factors. Journal of Vision, 8(4),31, 1-22. doi:10.1167/8.4.31.

Faubert, J., \& von Grünau, M. (1995). The influence of two spatially distinct primers and attribute priming on motion induction. Vision Research, 35, 3119-3130.

Hikosaka, O., Miyauchi, S., \& Shimojo, S. (1993a). Focal visual attention produces illusory temporal order and motion sensation. Vision Research, 33, 1219-1240. doi:10.1016/0042-6989 (93)90210-N

Hikosaka, O., Miyauchi, S., \& Shimojo, S. (1993b). Voluntary and stimulus-induced attention detected as motion sensation. Perception, 22, 517-526.

Hochberg, J., \& Brooks, V. (1978). The perception of motion pictures. In E. C. Carterette \& M. P. Friedman (Eds.), Handbook of perception (Vol. 10, pp. 259-304). New York, NY: Academic Press.

Hock, H. S., Gilroy, L., \& Harnett, G. (2002). Counter-changing luminance: A non-Fourier, nonattentional basis for the perception of single-element apparent motion. Journal of Experimental Psychology. Human Perception and Performance, 28, 92-112. doi:10.1037/0096-1523.28.1.93

Hock, H. S., \& Nichols, D. F. (2010). The line motion illusion: The detection of counterchanging edge and surface contrast. Journal of Experimental Psychology. Human Perception and Performance, 36, 781-796.

Hock, H. S., Schöner, G., \& Gilroy, L. (2009). A counterchange mechanism for the perception of motion. Acta Psychologica, 132, 1-21. doi:10.1016/j.actpsy.2009.06.006

Kanizsa, G. (1978). The polarization of gamma movements. Italian Journal of Psychology, 5, 265-285.

Lamme, V. A. F., Rodriguez-Rodriguez, V., \& Spekreijse, H. (1999). Separate processing dynamics for texture elements, boundaries and surfaces in primary visual cortex of the macaque monkey. Cerebral Cortex, 9, 406-413.

Marr, D., \& Nishihara, H. K. (1978). Representation and recognition of the spatial organization of three-dimensional Shapes. Proceedings of the Royal Society B, 211, 151-180.

Murray, S. O., Olshausen, B. A., \& Woods, D. L. (2003). Processing shape, motion and three-dimensional shapefrom-motion in the human cortex. Cerebral Cortex, 13, $508-516$.

Newsome, W. T., Mikami, A., \& Wurtz, R. H. (1986). Motion selectivity in macaque visual cortex: III. Psychophysics and physiology of apparent motion. Journal of Neurophysiology, 55, 1340 1351. 
Rodman, H. R., Gross, C. G., \& Albright, T. D. (1989). Afferent basis of visual response properties in area MT of the macaque: I. Effects of striate cortex removal. Journal of $\mathrm{Neu}$ roscience, 9, 2033-2050.

Rodman, H. R., Gross, C. G., \& Albright, T. D. (1990). Afferent basis of visual response properties in area MT of the macaque: II. Effects of superior colliculus removal. Journal of Neuroscience, 10, 1154-1164.

Saucer, R. T. (1953). The nature of perceptual processes. Science, 117, $556-558$

Sperling, G., \& Lu, Z.-L. (1998). A systems analysis of visual motion perception. In T. Watanabe (Ed.), High-level motion processing: Computational, neurobiological, and psychophysical perspectives (pp. 154-183). Cambridge: MA: MIT Press.
Steinman, R. M., Pizlo, Z., \& Pizlo, F. J. (2000). Phi is not beta, and why Wertheimer's discovery launched the Gestalt revolution: Mini review. Vision Research, 40, 2257-2264.

Tyler, C. W. (1973). Temporal characteristics in apparent movement: Omega movement vs. phi movement. Quarterly Journal of Experimental Psychology, 25, 171-181.

Verstraten, F. A. J., Cavanagh, P., \& Labianca, A. T. (2000). Limits of attentive tracking reveal temporal properties of attention. Vision Research, 40, 3651-3664. doi:10.1016/S0042-6989(00)00213-3

Wertheimer, M. (1912). Experimental studies of perceived movement (Experimentelle Studien über das Sehen von Bewegung). Zeitschrift für Psychologie und Physiologie der Sinnesorgane, 61, 161-265.

Zeki, S. M. (1974). Functional organization of a visual area in the posterior bank of the superior temporal sulcus of the rhesus monkey. The Journal of Physiology, 236, 549-573. 